\title{
Three Basic Antibiotics, Streptomycin, Roseomycin and No. 259 in Experi- mental Typhoid Infection
}

(Studies on the Antibiotic Substances from Actinomyces, 11th Report)

By

Ippei Nagao

(長尾一平)

From the Department of Bacteriology, Faculty of Medicine, Tohoku University, Sendai. Director: Prof. M. Kuroya

(Received for publication, November 4, 1952)

There are many cases of typhoid fever in our country, but no effective chemotherapeutic agents have been devised yet to treat this infection. The antibiotics obtained from streptomyces, such as streptomycin, ${ }^{12)}$ chloramphenicol, ${ }^{3)}$ aureomycin and terramycin,${ }^{4)}$ seem to be very beneficial against typhoid infections.

During the systematic study of the antagonistic actinomyces in our laboratory, streptomycin and many streptothricin-like substances were isolated and crystallized.5) The present study deals with activities of streptomycin and two streptothricin-like substances against Salmonella typhosa both in vitro and in experimental infection. (The experiment conducted in 1949).

\section{EXPERIMENTAL}

Materials and Methods

1) Streptomycin used was HCl-calcium chloride double salt, Merck.

2) Roseomycin ${ }^{6)}$ (No. $36 \mathrm{HCl}$-salt) was a representative substance of the so-called streptothricin type 1 (ST 1) of our laboratory, which was characterized in low toxicity, good diffusibility and low potency against gram positive organisms.

3) No. $259 \mathrm{HCl}-\mathrm{salt}^{5}$ ) was a representative substance of the so-called streptothricin type 2 (ST 2), and characterized in high potency, less diffusibility and relatively high toxicity.

In vitro activity was tested on one hand by serial dilution method with nutrient broth $(1 \%$ meat ext., $1 \%$ peptone and $0.3 \% \mathrm{NaCl}, \mathrm{pH}$ 7.5). The total volume of test fluid divided into each tube was $5 \mathrm{cc}$. One drop $(0.05 \mathrm{cc}$.) of 10 fold dilution of 24 hours broth culture of test 
TAB L E I

Activity Ratio of 3 Antibiotics against E. coli and S. typhosa. (Dilution Unit)

\begin{tabular}{ll|c|c}
\hline \multirow{2}{*}{ Antibiotics } & \multicolumn{2}{|c|}{ Test organisms } & $\begin{array}{c}\text { Activity ratio } \\
\text { S. typhosa: } \\
\text { E. coli }\end{array}$ \\
\cline { 2 - 4 } & $\begin{array}{c}\text { E. coli (Umezawa } \\
\text { strain) }\end{array}$ & $\begin{array}{c}\text { S. typhosa } \\
\text { (T-63 strain) }\end{array}$ & $\begin{array}{c}\text { (T) } \\
\text { a. Streptomycin }\end{array}$ \\
b. Roseomycin & $160^{*} \mathrm{u} / \mathrm{mg}$. & $20 \mathrm{u} / \mathrm{mg}$. & $1 / 1$ \\
c. No. 259 substance & 16 & 20 & $1 / 2$ \\
& 80 & 200 &
\end{tabular}

* Minimum amount required for complete inhibition

\section{TAB L E II}

Dilution Unitage of Streptomycin and Roseomycin Against 6 Strains of $S$. typhosa

\begin{tabular}{|c|c|c|c|c|c|c|c|c|c|c|c|}
\hline \multirow{3}{*}{ Strain tested } & \multicolumn{10}{|c|}{$\mu \mathrm{g}$ of the antibiotics contained in $1 \mathrm{cc}$. of broth } & \multirow{3}{*}{ 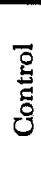 } \\
\hline & \multicolumn{5}{|c|}{ Streptomycin } & \multicolumn{5}{|c|}{ Roseomycin } & \\
\hline & $\frac{1}{10}$ & $\frac{1}{20}$ & $\frac{1}{40}$ & $\frac{1}{80}$ & $\frac{1}{100}$ & $\frac{1}{10}$ & $\frac{1}{20}$ & $\frac{1}{40}$ & $\frac{1}{80}$ & $\frac{1}{160}$ & \\
\hline No. 50 & - & - & \pm & + & + & - & - & + & + & + & H \\
\hline No. 53 & - & - & $H$ & $H$ & H & - & - & + & + & $H$ & \# \\
\hline No. 58 & - & - & - & + & $H$ & - & - & \pm & + & $H$ & H \\
\hline T-63 (U.S.A.) & - & - & + & + & + & - & - & \pm & + & + & $H$ \\
\hline No. $14 *$ & - & - & \pm & + & $H$ & - & - & \pm & + & $H$ & H \\
\hline Haryu-strain & - & - & + & $H$ & H & - & - & \pm & \pm & + & $H$ \\
\hline
\end{tabular}

$\mathrm{H}, \mathrm{H},+=$ visible growth, showing its grade. $\pm=$ incomplete inhibition. $-=$ complete inhibition. * Strains of $S$. typhosa preserved in the author's laboratory.

organisms, i.e S. typhosa and E. coli, were inoculated into each tube. Diffusion assay was conducted on the other according to the standard pulp disc assay of streptomycin.

In vivo activity was tested in mice weighing 15 to $23 \mathrm{~g}$., and challenged with $S$. typhosa (T-63 strain), suspended in 5\% mucin solution.

\section{Results}

As shown in Table $\mathrm{I}$, activity ratio of 3 antibiotics i.e. streptomycin, roseomycin and No. 259 against $E$. coli was 10:1:5, while that against S. typhosa was $1: 1: 10$. Although in vitro test, No. 259 seems to be most effective in such a manner, the preliminary test in vivo showed a contrary result. From this point of view, chiefly roseomycin and streptomycin 


\section{TAB LE III}

Efficiency of Streptomycin, Reseomycin, and No. 259 Substance in Protecting Mice against $S$. typhosa, Administered Intraperitoneally Immediately after Inoculation

\begin{tabular}{|c|c|c|c|c|c|c|c|c|c|c|c|c|c|c|}
\hline \multirow{2}{*}{$\begin{array}{c}\text { Animal } \\
\text { group }\end{array}$} & \multirow{2}{*}{$\begin{array}{l}\text { No. } \\
\text { of } \\
\text { mice }\end{array}$} & \multirow{2}{*}{$\begin{array}{c}\text { mg of organisms per } \\
0.5 \mathrm{cc} \text {. in mucin solu- } \\
\text { tion injected intra- } \\
\text { peritoneally }\end{array}$} & \multirow{2}{*}{$\begin{array}{l}\text { Single } \\
\text { dosage } \\
\text { (mg.) }\end{array}$} & \multicolumn{9}{|c|}{$\begin{array}{l}\text { No. of mice that } \\
\text { died on day }\end{array}$} & \multirow{2}{*}{ คี } & \multirow{2}{*}{ 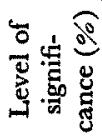 } \\
\hline & & & & 1. & 2. & 3. 4 & 5. & 6. & 7. & 8. & 9. & 10. & & \\
\hline Control & $\begin{array}{l}\mathbf{5} \\
\mathbf{5} \\
\mathbf{5} \\
\mathbf{5}\end{array}$ & $\begin{array}{l}10^{-4} \\
10^{-5} \\
10^{-6} \\
10^{-7}\end{array}$ & & $\begin{array}{l}5 \\
4 \\
3 \\
1\end{array}$ & $\begin{array}{l}1 \\
0 \\
0\end{array}$ & $\begin{array}{l}2 \\
1\end{array}$ & 0 & 0 & 0 & 0 & 0 & 0 & $\begin{array}{l}5 / 5 \\
5 / 5 \\
5 / 5 \\
2 / 5\end{array}$ & \\
\hline $\begin{array}{l}\text { Strepto- } \\
\text { mycin }\end{array}$ & $\begin{array}{l}5 \\
5 \\
5\end{array}$ & $\begin{array}{l}10^{-6} \\
10^{-6} \\
10^{-6}\end{array}$ & $\begin{array}{l}0.03 \\
0.05 \\
0.1\end{array}$ & $\begin{array}{l}1 \\
0 \\
0\end{array}$ & \begin{tabular}{l|l}
0 & \\
0 & \\
0 &
\end{tabular} & \begin{tabular}{l|l}
0 & 0 \\
0 & 0 \\
0 & 0
\end{tabular} & $\begin{array}{l}0 \\
0 \\
0\end{array}$ & $\begin{array}{l}0 \\
0 \\
0\end{array}$ & $\begin{array}{l}0 \\
0 \\
0\end{array}$ & $\begin{array}{l}0 \\
0 \\
0\end{array}$ & $\begin{array}{l}0 \\
0 \\
0\end{array}$ & $\begin{array}{l}0 \\
0 \\
0\end{array}$ & $\begin{array}{l}1 / 5 \\
1 / 5 \\
0 / 5\end{array}$ & $\begin{array}{l}2.4 \\
2.4 \\
0.4\end{array}$ \\
\hline $\begin{array}{c}\text { No. } 259 \\
\text { substance }\end{array}$ & $\begin{array}{l}5 \\
5 \\
5\end{array}$ & $\begin{array}{l}10^{-6} \\
10^{-6} \\
10^{-6}\end{array}$ & $\begin{array}{l}0.06 \\
0.1 \\
0.2\end{array}$ & $\begin{array}{l}1 \\
0 \\
0\end{array}$ & $\begin{array}{l}0 \\
0 \\
1\end{array}$ & \begin{tabular}{l|l}
0 & 0 \\
1 & 0 \\
0 & 0
\end{tabular} & $\begin{array}{l}0 \\
0 \\
0\end{array}$ & $\begin{array}{l}0 \\
0 \\
0\end{array}$ & $\begin{array}{l}0 \\
0 \\
0\end{array}$ & $\begin{array}{l}0 \\
0 \\
0\end{array}$ & $\begin{array}{l}1 \\
0 \\
0\end{array}$ & $\begin{array}{l}0 \\
0 \\
0\end{array}$ & $\begin{array}{l}2 / 5 \\
1 / 5 \\
1 / 5\end{array}$ & $\begin{array}{l}8.3 \\
2.4 \\
2.4\end{array}$ \\
\hline $\begin{array}{l}\text { Roseo- } \\
\text { mycin }\end{array}$ & $\begin{array}{l}5 \\
5 \\
5\end{array}$ & $\begin{array}{l}10^{-6} \\
10^{-6} \\
10^{-6}\end{array}$ & $\begin{array}{l}0.075 \\
0.125 \\
0.25\end{array}$ & $\begin{array}{l}0 \\
0 \\
0\end{array}$ & $\begin{array}{l}1 \\
0 \\
0\end{array}$ & \begin{tabular}{l|l}
0 & \multicolumn{1}{c}{} \\
0 & 0 \\
0 &
\end{tabular} & $\begin{array}{l}0 \\
0 \\
0\end{array}$ & $\begin{array}{l}0 \\
0 \\
0\end{array}$ & $\left|\begin{array}{l}0 \\
0 \\
0\end{array}\right|$ & $\left|\begin{array}{l}0 \\
0 \\
0\end{array}\right|$ & $\begin{array}{l}0 \\
0 \\
0\end{array}$ & $\begin{array}{l}0 \\
0 \\
0\end{array}$ & $\begin{array}{l}1 / 5 \\
0 / 5 \\
0 / 5\end{array}$ & $\begin{array}{l}2.4 \\
0.4 \\
0.4\end{array}$ \\
\hline
\end{tabular}

TABLE IV

Efficiency of Streptomycin and Roseomycin in Protecting Mice against $S$. typhosa*

\begin{tabular}{|c|c|c|c|c|c|c|c|c|c|c|c|c|c|c|}
\hline \multirow{2}{*}{ 氧骂 } & \multirow{2}{*}{ 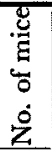 } & \multirow{2}{*}{ 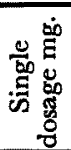 } & \multirow{2}{*}{$\begin{array}{l}\text { Administration } \\
\text { method }\end{array}$} & \multicolumn{9}{|c|}{$\begin{array}{l}\text { No. of mice that } \\
\text { died on day }\end{array}$} & \multirow{2}{*}{ 吾苞 } & \multirow{2}{*}{ 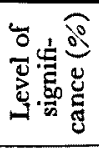 } \\
\hline & & & & 1. & 2. & 3. & & 6 & 7 & 8 & 9. & 10. & & \\
\hline $\begin{array}{c}\text { Cont- } \\
\text { rol }\end{array}$ & 15 & & & 9 & 4 & 0 & 0 & 0 & 0 & 0 & 0 & 0 & $13 / 15$ & \\
\hline \multirow{4}{*}{ 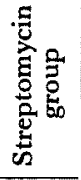 } & 10 & 0.05 & Intraperitoneal & 0 & 5 & 0 & 0 & 0 & 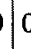 & 0 & 0 & 0 & $6 / 10$ & 14.7 \\
\hline & 5 & 0.2 & Intramuscular & 2 & 0 & 0 & 0 & c & 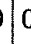 & 0 & 0 & 0 & $2 / 5$ & 7.3 \\
\hline & 5 & 0.2 & Intravenous & 1 & 1 & 0 & 0 & c & ) & 0 & 0 & 0 & $2 / 5$ & 7.3 \\
\hline & 5 & 0.4 & Intramuscular & 0 & 0 & 0 & 0 & 0 & 0 & 0 & 0 & 0 & $0 / 5$ & 0.14 \\
\hline \multirow{4}{*}{ 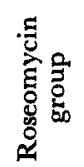 } & 10 & 0.05 & Intraperitoneal & 3 & 3 & 0 & 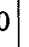 & 10 & & 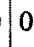 & & 0 & $7 / 10$ & 24.8 \\
\hline & 5 & 0.2 & Intramuscular & 2 & 0 & 0 & 0 & c & & 0 & & 0 & $2 / 5$ & 7.3 \\
\hline & 5 & 0.2 & Intravenous & 1 & 0 & 1 & 0 & c & & 0 & & 0 & $2 / 5$ & 7.3 \\
\hline & 5 & 0.4 & Intramuscular & 0 & $0 \mid$ & 0 & 9 & 0 & & & & 0 & $0 / 5$ & 0.14 \\
\hline
\end{tabular}

* Strain: T-63 (U.S.A.)., Dose of organisms: $10^{-6} \mathrm{mg}$. in $0.5 \mathrm{cc}$. of $5 \%$ mucin solution. Inoculations were made intraperitoneally. Drugs were administered 1 hour after the infection.

were selected further to test on their activities in vitro against 6 strains of $S$. typhosa. Almost equivalent activity was indicated with both anti- 
TABLE V

Diffusion Activity of 3 Antibiotics against $S$. typhosa T-63

\begin{tabular}{|c|c|c|c|c|c|c|c|c|c|c|c|c|c|c|c|c|c|c|}
\hline \multirow{2}{*}{$\begin{array}{l}\mu g \text { of } \\
\mathrm{HCl} \\
\text { salt }\end{array}$} & \multicolumn{6}{|c|}{ Streptomycin } & \multicolumn{6}{|c|}{ Roseomycin } & \multicolumn{6}{|c|}{ No. 259 substance } \\
\hline & I & II & III & IV & V & Aver. & J & II & III & IV & V & Aver. & I & II & III & IV & $\mathbf{V}$ & Aver. \\
\hline 31 & 17.0 & 17.0 & 16.4 & 16.7 & 16.4 & 16.70 & 16.9 & 17.0 & 17.0 & $17.5 \mid$ & 16.3 & 16.94 & 12.3 & 11.4 & 12.6 & 12.2 & 12.3 & 12.16 \\
\hline 63 & 19.2 & 18.7 & 18.9 & 17.6 & 18.6 & 18.80 & $\mid 18.9$ & 18.7 & 18.6 & 19.4 & 18.4 & 18.80 & 14.4 & 13.9 & 14.6 & 14.3 & 14.6 & 14.36 \\
\hline 125 & 21.4 & 20.7 & 21.0 & 20.8 & 21.1 & 21.00 & 21.4 & 20.8 & 20.5 & 21.6 & 21.0 & 21.06 & 16.2 & 15.8 & 16.2 & 16.1 & 16.3 & 16.12 \\
\hline 250 & 23.2 & 22.0 & 1033 & 22.4 & 23.0 & 22.78 & 23.2 & 22.6 & 22.5 & 23.4 & 22.0 & 22.74 & 17.9 & 17.7 & 17.4 & 17.4 & 17.5 & 17.58 \\
\hline 500 & 24.5 & 23.3 & 24.4 & 24.2 & 24.2 & 24.12 & 24.2 & 23.8 & 23.6 & 24.7 & 23.7 & 24.00 & 18.7 & 18.6 & 18.4 & 18.7 & 18.9 & 18.66 \\
\hline
\end{tabular}

The figures indicate the diameter of inhibition zones assayed by pulp disc method in mm.

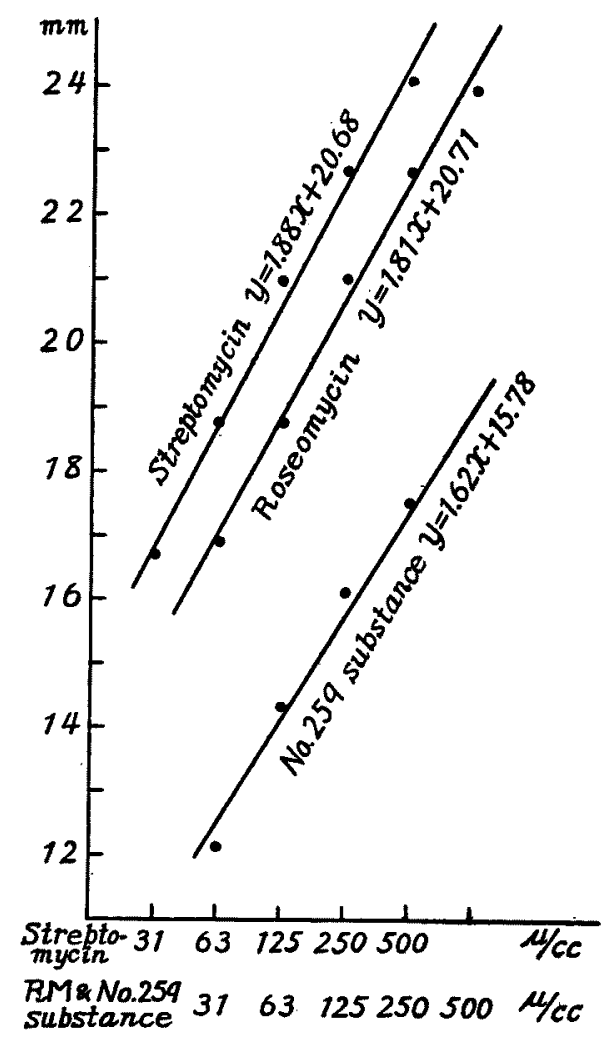

Fig. 1. Diffusibilities of 3 basic antibiotics

biotics, as shown in Table II.

As shown in Table III, in vivo activities were compared with 3 antibiotics. The antibiotics were administered immedately after infection. 
As the result almost same activity was proved with these 3 , so the high activity of No. 259 in vitro was not confirmed in vivo.

The second in vivo test was conducted with streptomycin and roseomycin both administered 1 hour after the infection. The administration was made through 3 routes, as shown in Table IV. 8 times of dosage was required with both antibiotics, when compared with the former experiment. From the above experiment, it was revealed, that, though the in vitro activity of roseomycin against gram positive organisms is low, it has a remarkable effectiveness against gram negative organisms in vitro and in vivo.

Finally, diffusion activity of 3 antibiotics against typhoid bacilli was compared with each other, as shown in Table V and Fig. 1. Least diffusibillity of No. 25.9 substance appeared to be closely correlated with the in vivo inactivity of it.

\section{SUMMARY}

The activities of 3 antibiotics, streptomycin, roseomycin and No. 259 substance were tested against $S$. typhosa both in vitro and in vivo. The effectiveness of streptomycin and roseomycin has been proved especially in vivo.

\section{References}

1) Robinson, H. P., Proc. Soc. Exp. Biol. and Med., 1944, 57, 226.

2) Keefer, C. S. et al., J. Amer. Med. Ass., 1946, 132, 4; do, 1946, 132, 70.

3) Margatroyd, F., Brit. Med. J., 1949, I, 851.

4) Mikamo, Y., Nippon Rinsho (Japanese Clin. J.), 1948, 6, 179.

5) Kuroya, M., Ishida, N., Katagiri, K., Konno, J., Kikuchi, M., and Mizuguchi, R., Tohoku J. Exp. Med., 1952, 55, 289.

6) Ishida, N., ibid., 1953, 58, 153. 